\title{
Tribología de nuevas capas autolubricantes producidas mediante PVD
}

\author{
J.I. Oñate, M. Brizuela*, A. García-Luis", I. Braceras", S. Pacheco* y J.L. Viviente* \\ Resumen Las capas de $\mathrm{MoS}_{2}$ depositadas mediante PVD tienen baja fricción en vacío pero se \\ degradan con facilidad al aire, especialmente en presencia de humedad. Este trabajo \\ describe el desarrollo de nuevas capas de $\mathrm{MoS}_{\mathrm{x}}$ modificadas con WC. En ensayos de "bola \\ sobre disco" a 0,75 GPa, las capas presentaron una fricción muy baja y estable $(<0,04)$ en \\ vacío. Se obtuvo una durabilidad superior a 1 millón de ciclos, mostrando un desgaste de \\ $1,3 \times 10^{-16} \mathrm{~m}^{3} / \mathrm{Nm}$, mejorado respecto a otras capas de $\mathrm{MoS}_{\mathrm{x}}$. El coeficiente de fricción \\ aumentó a 0,15 y la durabilidad disminuyó hasta 1 a $3 \times 10^{5}$ ciclos en aire hasta $70 \% \mathrm{HR}$. \\ El análisis superficial mostró ratios de S/Mo superiores a 1,2, con menos del 2 \% de oxígeno, \\ demostrando carácter lubricante.
}

Palabras clave Capas autolubricantes. $\mathrm{MoS}_{2}$. PVD. Tribología. Desgaste. Fricción.

\section{Tribology of new solid lubricating films produced by PVD}

\begin{abstract}
MoS}_{2}$ coatings, deposited by PVD show a low friction under vacuum conditions, but degrade easily under atmospheric conditions. This work describes the development of a new type of $\mathrm{MoS}_{\mathrm{x}}$ coating, modified with WC. These coatings presented a very low and stable friction coefficient lower than 0.04 when tested under vacuum at $0.75 \mathrm{GPa}$ in a ball on disc tribometer. Durability was higher than 1 million wear cycles, showing a wear rate of $1.310^{-16} \mathrm{~m}^{3} / \mathrm{Nm}$. When tested under atmospheric conditions and up to $70 \% \mathrm{RH}$, friction increased to 0.15 and durability reached a lower value of up to $3 \times 10^{5}$ cycles. Surface analyses in these coatings showed S/Mo ratios higher than 1.2 and with oxygen levels lower than $2 \%$, demonstrating the lubricant character of the coating.
\end{abstract}

Keywords Solid lubricant coatings. $\mathrm{MoS}_{2}$. PVD. Tribology. Wear. Friction.

\section{INTRODUCCIÓN}

En los últimos años se ha prestado una creciente atención al desarrollo y mejora de sistemas de lubricación sólida, como consecuencia de presiones medioambientales sobre los lubricantes líquidos que se utilizan en numerosos procesos metalúrgicos. El requisito para estos lubricantes sólidos es que mantengan coeficientes de rozamiento muy bajos en las condiciones operativas de producción, transmitiendo, por tanto, menores tensiones a las herramientas. Adicionalmente, la eliminación o reducción de los lubricantes líquidos conlleva unos ahorros importantes en líneas de limpieza y el coste medioambiental asociado.

Uno de los lubricantes sólidos más utilizados, principalmente en el ambiente aeroespacial, ha sido el bisulfuro de molibdeno $\left(\mathrm{MoS}_{2}\right)^{[1]}$. Este material presenta unas excelentes propiedades tribológi- cas en vacío, sin embargo, en condiciones atmosféricas, su comportamiento se ve sensiblemente afectado, debido a su oxidación ${ }^{[2}$ y 3]. El reto ha sido, en consecuencia, la mejora y el desarrollo de nuevas capas basadas en este compuesto, manteniendo sus excelentes propiedades en vacío, pero con una mayor resistencia a la oxidación y operación en condiciones de alta humedad ${ }^{[4}$ y 5$]$. Por medio de la aleación de estas capas con ciertos metales como por ejemplo el titanio, se ha conseguido cierto éxito, debido a la afinidad de este elemento con el oxígeno, evitando la formación de otros óxidos perjudiciales ${ }^{[4,6 \text { y } 7]}$. Las aplicaciones de este lubricante en mecanismos espaciales pueden beneficiarse de esta mejora, ya que no sólo exhibirían un buen comportamiento en vacío, sino también al aire y a mayores porcentajes de humedad, sin una degradación excesiva. Los ensayos de calificación previos al vuelo se podrían realizar sin las 
actuales limitaciones del $\mathrm{MoS}_{2}$, que obligan a la utilización del vacío o de atmósferas inertes.

La motivación de este trabajo ha sido, por tanto, el desarrollo de un nuevo tipo de lubricante sólido, basado en el $\mathrm{MoS}_{2}$ con una mayor durabilidad e inferior degradación bajo condiciones atmosféricas, lo que permitiría su aplicación no sólo en el ambiente espacial sino en determinadas herramientas de corte y conformado metálico. Finalmente, las capas seleccionadas se enviarán, para su ensayo mediante discos y rodamientos, al laboratorio TriboLAB que se instalará en el exterior del Laboratorio Columbus de la ESA en la Estación Espacial Internacional (ISS) ${ }^{[8]}$.

\section{PROCEDIMIENTO EXPERIMENTAL}

Las capas de $\mathrm{MoS}_{\mathrm{x}}$ modificadas con WC se depositaron sobre discos pulidos de $45 \mathrm{~mm}$ de diámetro y $5 \mathrm{~mm}$ de espesor, de acero inoxidable martensítico AISI $440 \mathrm{C}$ templado y revenido a una dureza de $57 \pm 1 \mathrm{HRc}$ y con un acabado superficial de $0,01 \mu \mathrm{m} \mathrm{Ra}$. Para la producción de las capas se utilizó un equipo de PVD CemeCon CC800/8, mediante la técnica de magnetron sputtering partiendo de 4 cátodos ( 1 a 2 de $M_{o} S_{2}$ y 1 a 2 cátodos de WC) en una descarga de argón a una presión de 0,5 a 0,7 Pa. El espesor de las capas depositadas fue de 1 a $1,5 \mu \mathrm{m}$.

La microdureza de las capas se midió con un equipo FISCHERSCOPE H100 que permite la medición dinámica con un indentador Vickers convencional a cargas muy bajas, minimizando la influencia del material base, utilizando un procedimiento previamente descrito ${ }^{[9]}$. La composición y estudio del estado químico de los elementos presentes en las capas se realizó en un espectrómetro MICROLAB MKII de VG mediante la técnica de Small Area X-ray Photoelectron Spectroscopy (SAXPS) combinada con erosión de iones $\mathrm{Ar}^{+}$a $4 \mathrm{keV}$. Las muestras se excitaron con radiación $\mathrm{Al} \mathrm{K \alpha}$ a $34 \mathrm{~mA}$ y $13 \mathrm{kV}$ a una presión inferior a $2 \times 10^{-9} \mathrm{mbar}$ (sin argón). De cada muestra se extrajeron espectros detallados de Mo 3d, S 2p, W 4f, C 1s, O 1s, Fe 2p y Cr 2p.

Los ensayos de desgaste y fricción se realizaron con dos tipos de tribómetros de geometría "bola sobre disco". El primero, un equipo de desgaste CSEM, para la realización de ensayos en vacío $\left(<10^{-5} \mathrm{mbar}\right)$, con cargas de 5 y $10 \mathrm{~N}$, con una bola de acero AISI $440 \mathrm{C}$ de $6 \mathrm{~mm}$ diámetro a una velocidad constante de $0,5 \mathrm{~m} / \mathrm{s}$. La máxima presión de contacto estimada en estas condiciones es de
0,75 y 0,95 GPa respectivamente. Adicionalmente, se utilizó un equipo FALEX ISC 320 para la obtención de datos comparativos en condiciones atmosféricas, con idénticas cargas y velocidades, pero realizando los ensayos tribológicos en condiciones de humedad relativa del 40 al $80 \%$ RH. Todos los ensayos se realizaron por triplicado, hasta alcanzar un coeficiente de rozamiento máximo de 0,2. El volumen de desgaste y la morfología del mismo se evaluaron tanto en discos como en bolas por medio de perfilometría láser y microscopio electrónico de barrido (SEM), respectivamente.

\section{RESULTADOS Y DISCUSIÓN}

\subsection{Análisis superficial mediante XPS}

El análisis mediante XPS de estas capas posee la limitación del decapado preferencial de azufre y carbono, haciendo difícil la determinación de la concentración de los elementos. A pesar de ello, el perfil de su intensidad en función de la profundidad mostró que se obtenía una relación aproximadamente fija en la zona uniforme del recubrimiento.

La figura 1 muestra los espectros Mo $3 \mathrm{~d}$ y W $4 \mathrm{f}$ de una capa $\mathrm{MoS}_{\mathrm{x}} / \mathrm{WC}$ (Ref. 25956) a diferentes tiempos de decapado. En esta figura se incluyen los espectros del $\mathrm{MoS}_{2}$ y de WC-Co como referencia, en la parte inferior. Los espectros de las regiones Mo 3d, S 2p, W 4f y C 1s a los tiempos de decapado seleccionados muestran que se da una elevada contribución de enlaces Mo-S y W-C, así como una baja contribución del enlace C-C, sin evidencia clara de W-S o el posible Mo-C. Parece deducirse que las fases de Mo-S y W-C permanecen independientes $y$, a pesar del decapado preferencial del azufre, la relación S/Mo en la capa puede calcularse de acuerdo a la fórmula expresada de Baker et al. ${ }^{[10]}$, obteniéndose un valor de 1,55 que anticipa para la capa un comportamiento lubricante.

\subsection{Tribología y propiedades mecánicas}

Los ensayos de microindentación dinámica realizados sobre la capa de $\mathrm{MoS}_{\mathrm{x}} / \mathrm{WC}$ mostraron una dureza bajo carga de $5.179 \mathrm{~N} / \mathrm{mm}^{2}$ a $10 \mathrm{mN}$ y una recuperación elástica del 34,29\%. Estos valores de baja dureza son propios de una capa de este material lubricante.

Existen diversos tipos de ensayos de desgaste que pueden realizarse sobre materiales recubiertos o tratados superficialmente ${ }^{[11]}$, si bien los ensayos de la geometría utilizada en el presente trabajo 

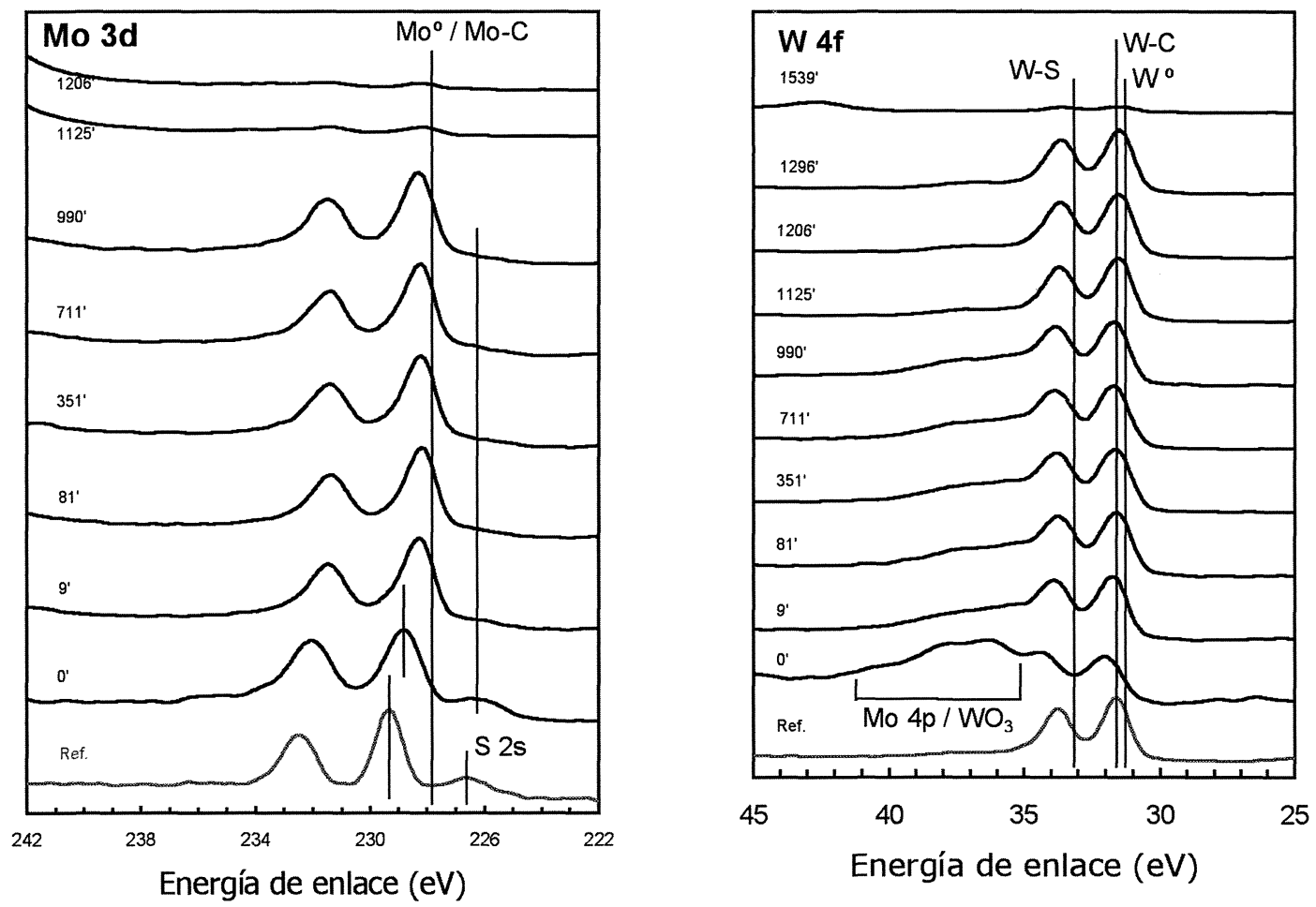

Figura 1. Espectros XPS de Mo 3d (izqda) y W $4 f$ (dcha) de la capa MoS $/$ WC (Ref. 25956) a diferentes tiempos de decapado. Se incluye un espectro de referencia en la parte inferior, de $\mathrm{MoS}_{2}$ en el Mo $3 \mathrm{~d}$ y de WC-Co en el W $4 \mathrm{f}$.

Figure 1. Mo 3d (left) and W 4f (right) XPS spectra from MoS $/$ WC film (Ref. 25956) at different pickling times. Reference spectra are included in the lower part, from $\mathrm{MoS}_{2}$ in the Mo $3 d$ and from WC-Co in the W $4 f$.

(bola sobre disco) son los más comunes para la evaluación de capas autolubricantes ${ }^{[12]}$.

Los resultados de fricción y desgaste en vacío de estas capas pueden verse en la tabla I y la figura 2, mientras que en condiciones atmosféricas se muestran en la tabla II y la figura 3. Estas tablas resumen los resultados de tribología de una capa representativa, incluyendo datos del coeficiente de rozamiento medio, la velocidad de desgaste del dis-

Tabla I. Resultados representativos de desgaste en vacío (Ref. MoSx/WC 25956). Condiciones: $5 \mathrm{~N} ; 0,5 \mathrm{~m} / \mathrm{s}$; bola de acero AISI $440 C$ de $6 \mathrm{~mm}$ diámetro

Table I. Representative wear test results under vacuum (Ref. MoS $/$ WC 25956). Testing conditions: $5 \mathrm{~N} ; 0,5 \mathrm{~m} / \mathrm{s}$; $6 \mathrm{~mm}$ diameter AISI $440 \mathrm{C}$ steel ball

\begin{tabular}{lrcc}
\hline $\begin{array}{l}\text { Ensayo } \\
\text { Ref. }\end{array}$ & $\begin{array}{c}\text { Duración } \\
\left(\mathbf{N}^{\circ} \text { ciclos }\right)\end{array}$ & $\begin{array}{c}\text { Velocidad desgaste } \\
\text { disco }\left(\mathrm{mm}^{3} / \mathrm{Nm}\right)\end{array}$ & $\begin{array}{c}\text { Fricción } \\
\mu_{\text {media }}\end{array}$ \\
\hline AM 1014 & 657.260 & $2,4 \times 10^{-7}$ & 0,04 \\
AM 1015 & 1.196 .049 & $1,3 \times 10^{-7}$ & 0,03 \\
AM 1024 & 609.563 & $9,5 \times 10^{-8}$ & 0,02 \\
\hline
\end{tabular}

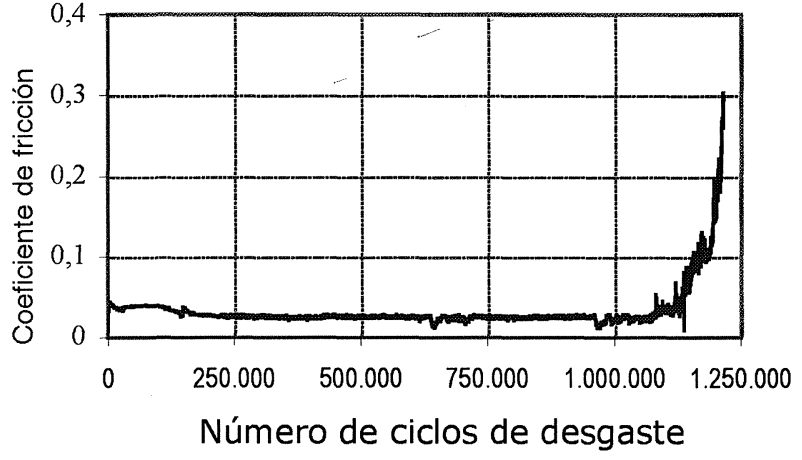

Figura 2. Curva de rozamiento característica para una capa de $\mathrm{MoS}_{x} / \mathrm{WC}$, ensayada en vacío a 0,75 GPa.

Figure 2. Characteristic friction curve for a MoS ${ }_{x} / W C$ film, tested under vacuum at 0,75 GPa.

co recubierto y la durabilidad de la capa en ciclos de desgaste.

La capa de $\mathrm{MoS}_{\mathrm{x}} / \mathrm{WC}$ muestra un coeficiente de rozamiento muy bajo en vacío, de 0,02 a 0,04, característico del $\mathrm{MoS}_{2}$ producido mediante pulverización catódica (Tabla I). La durabilidad de esta capa a 0,75 GPa fue de 1,2 millones de ciclos, que compara muy favorablemente con las capas de 
Tabla II. Resultados de desgaste representativos obtenidos en condiciones atmosféricas. (Ref. MoS $/$ WC 25956).

Condiciones de ensayo: $5 \mathrm{~N}, 0,5 \mathrm{~m} / \mathrm{s}$, bola acero AISI

$440 \mathrm{C}$ de $6 \mathrm{~mm}$ diámetro, humedad relativa del 40 a $60 \%$

Table II. Representative wear test results under atmospheric conditions. (Ref. MoS $/$ WC 25956). Testing conditions:

$5 \mathrm{~N} ; 0,5 \mathrm{~m} / \mathrm{s} ; 6 \mathrm{~mm}$ diameter AISI 440C steel ball; 40 to $60 \%$ relative humidity

\begin{tabular}{lcccc}
\hline $\begin{array}{c}\text { \% Humedad } \\
\text { en ensayo }\end{array}$ & $\begin{array}{c}\text { Duración } \\
\text { (ciclos) }\end{array}$ & $\begin{array}{c}\text { Velocidad } \\
\text { desgaste } \\
\text { disco } \\
\left(\mathrm{mm}^{3} / \mathrm{Nm}\right)\end{array}$ & $\begin{array}{c}\text { Máxima } \\
\text { fricción } \\
\mu_{\max }\end{array}$ & $\begin{array}{c}\text { Fricción } \\
\text { media } \\
\mu_{\text {media }}\end{array}$ \\
\hline $40 \% \mathrm{HR}$ & 322.169 & $3,1 \times 10^{-7}$ & 0,15 & 0,07 \\
$50 \% \mathrm{HR}$ & 238.541 & $4,7 \times 10^{-7}$ & 0,12 & 0,09 \\
$60 \% \mathrm{HR}$ & 447.622 & $1,3 \times 10^{-7}$ & 0,19 & 0,15 \\
\hline
\end{tabular}

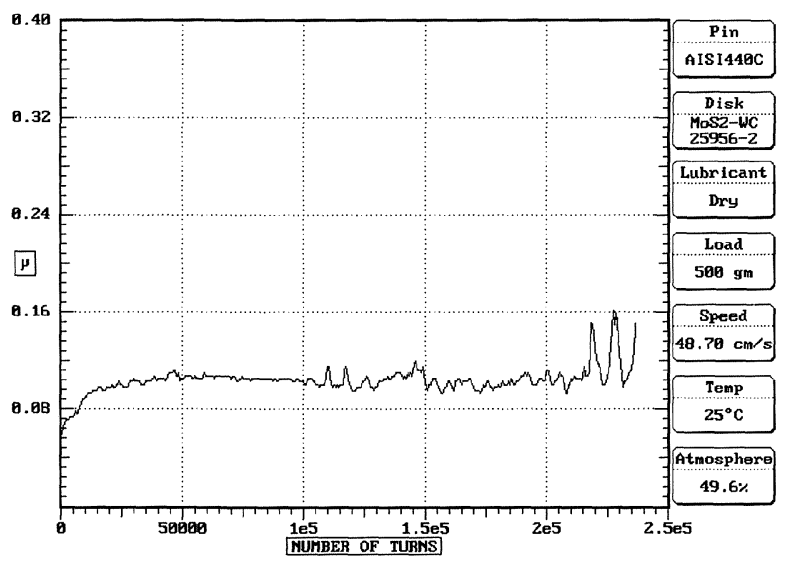

Figura 3. Variación del rozamiento en una capa de $\mathrm{MoS}_{\mathrm{x}} / \mathrm{WC}$ ensayada en aire a $50 \%$ humedad relativa.

Figure 3. Friction curve from a MoS $x /$ WC film tested under air at $50 \%$ relative humidity.

$\mathrm{MoS}_{2}$ aleadas con titanio ensayadas en condiciones idénticas ${ }^{[4]}$, y con lubricantes sólidos convencionales de acuerdo a datos publicados en el Space Tribology Handbook ${ }^{[12]}$. Al ensayarse la capa a una mayor presión de contacto de 0,95 GPa $(10 \mathrm{~N}$ de carga), su vida disminuyó a 150.000 ciclos, considerándose, aún éste, un resultado notable.

La evolución del coeficiente de rozamiento fue muy similar en la mayoría de los casos, con valores algo superiores al inicio, que pronto disminuían a valores inferiores y más estables, después de un periodo breve de rodadura ocasionado por la transferencia de material lubricante del disco a la superficie de la bola. Una vez que las propiedades lubricantes dejaron de existir en el contacto, el ro- zamiento aumentó hasta alcanzar el valor máximo de 0,2 del ensayo y éste se abortó. La transición de una fricción baja a otra más alta no fue abrupta en ningún caso. Un ejemplo de la variación del coeficiente de rozamiento de la capa $\mathrm{MoS}_{\mathrm{x}} / \mathrm{WC}$ puede verse en la figura 2.

Mediante observación en el SEM pudo apreciarse que la zona de desgaste en el disco no presentaba marcas abrasivas y el polvo de desgaste se acumulaba en los bordes del surco. El análisis efectuado en la superficie de contacto en la bola corroboró la transferencia de material de la capa, que explica la baja fricción obtenida. En este caso, la presencia de una fase dura y resistente al desgaste como el WC no parece degradar las propiedades lubricantes de la capa, extendiendo su durabilidad.

En la literatura no existen muchos datos de tribología en vacío de capas de $\mathrm{MoS}_{2}$ modificadas con metales. Pueden citarse, entre otros, los trabajos de Hinterman et al. ${ }^{[13]}$, Spalvins ${ }^{[14]}$ y más recientemente Hilton et al. ${ }^{[15]}$. En la mayoría de los casos, se realizó énfasis en desarrollar una capa lubricante con mayor resistencia a la humedad y, por tanto, con mejor comportamiento tribológico al aire. Adicionalmente, estas capas se depositaron utilizando la técnica de pulverización catódica con radiofrecuencia (rf) y produciendo en la mayoría de los casos una estructura dúplex compuesta de una capa delgada densa y con buena adherencia seguida de una estructura columnar, que es sólo válida para desgaste en vacío y en medio ambiente sin humedad.

Uno de los grupos más activos en los últimos años en el desarrollo de estas capas, modificadas con una variedad de elementos (ej. Ti, Cr, W, Zr etc.), ha sido Teer et al. ${ }^{[5,7 \text { y } 16]}$. Su objetivo ha sido el desarrollo de capas lubricantes para su aplicación en herramientas y han logrado depositar capas más duras y resistentes al desgaste en condiciones ambientales, sin ensayarlas en condiciones de vacío.

Los autores del presente trabajo también han demostrado en una publicación reciente ${ }^{[4]}$ que capas de $\mathrm{MoS}_{\mathrm{x}}$ modificadas con titanio exhiben una fricción muy baja y estable, de 0,07 a 0,1 , bajo condiciones atmosféricas y humedades relativas por debajo del 50-60\%. Por encima de esos niveles de humedad la capa se degrada más rápidamente, subiendo el rozamiento a niveles de 0,2 a 0,6 en condiciones de desgaste idénticas a las utilizadas en el presente trabajo.

Una de las motivaciones de este trabajo ha sido el desarrollo de capas no sólo con buen comportamiento en vacío sino también al aire, para facilitar 
los ensayos de calificación de mecanismos espaciales previos al vuelo. Algunos resultados de los ensayos realizados en condiciones ambientales se resumen en la tabla II y en la figura 3 .

Los resultados de estos ensayos son muy prometedores y se comparan favorablemente con los obtenidos por Matsumoto y Suzuki ${ }^{[17]}$ cuando éstos ensayan el $\mathrm{MoS}_{2}$ puro bajo diferentes condiciones de humedad, sin embargo no se puede realizar una comparación directa con los datos de Renevier et. al. ${ }^{[16]}$, ya que las condiciones de ensayo fueron muy diferentes.

Comparando estos resultados en condiciones atmosféricas y en vacío (Tabla I y Fig. 2), la durabilidad obtenida hasta un $60 \%$ de humedad relativa se puede considerar muy buena. El coeficiente de rozamiento medio aumenta de 0,07 a 0,15 al incrementar la humedad del 40 al $60 \%$, pero la alta durabilidad y la baja velocidad de desgaste en el disco recubierto parecen indicar que los ensayos de calificación en tierra de mecanismos espaciales podrían realizarse en aire con menores riesgos de degradación.

La figura 3 muestra la variación característica del rozamiento medio de una capa $\mathrm{MoS}_{\mathrm{x}} / \mathrm{WC}$ en función del número de ciclos de desgaste en un ensayo a $50 \%$ de humedad relativa. Estos resultados son similares a los obtenidos por otros investigadores con capas aleadas con otros elementos ${ }^{[16]}$, pero tienen un mejor comportamiento al compararlos con capas de $\mathrm{MoS}_{2}$ convencionales ${ }^{[18]}$, aunque en este último caso los autores indicaban que las capas tenían una baja concentración de azufre.

\section{CONCLUSIONES}

- Se han depositado capas de $\mathrm{MoS}_{\mathrm{x}} / \mathrm{WC}$ por medio del PVD (magnetron sputtering) que presentan buenas propiedades tribológicas, bajo vacío y en condiciones atmosféricas a diferentes grados de humedad.

- La tribología en vacío de estas capas muestra que la durabilidad a 0,75 GPa puede llegar hasta 1,2 millones de ciclos de desgaste, siendo éste un valor superior a lo publicado para capas de $\mathrm{MoS}_{2}$ convencionales. Esta mejora en la durabilidad va acompañada de una fricción reducida de 0,02 a 0,04. Tal como ocurre con los lubricantes sólidos convencionales, existe transferencia de material.

- Las capas de $\mathrm{MoS}_{\mathrm{x}} / \mathrm{WC}$ tienen un buen comportamiento tribológico bajo condiciones atmosféricas, a diferentes niveles de humedad (de
40 al $60 \% \mathrm{RH}$ ), mostrando una durabilidad tan alta como 450.000 ciclos y una fricción media de 0,07 a 0,15 .

- El W en la capa se encuentra principalmente en forma carbídica (W-C), no hay evidencia de enlaces de W-S.

- El buen comportamiento tribológico de estas capas se debe, principalmente, a la presencia de enlaces Mo-S y al bajo contenido de oxígeno en la capa. La presencia de la fase WC en la matriz de $\mathrm{MoS}_{\mathrm{x}}(\mathrm{x}>1,2)$ proporciona una velocidad de desgaste baja sin perjudicar una fricción reducida, característica de una capa autolubricante.

\section{Agradecimientos}

Los autores agradecen la cofinanciación de la DG Investigación del Ministerio de Ciencia y Tecnología (proyecto MAT2001-1399-C02-02) y del Departamento de Educación, Universidades e Investigación del Gobierno Vasco (proyecto PI2001-12).

\section{REFERENCIAS}

[1] T. SpalVINS, NASA TMX 67832 (1971).

[2] R.L. FusARO, NASA TP-1343 (1978).

[3] I.L. Singer, S. FAYeulle y P.D. EhNi, Wear 195 (1996) 7-20.

[4] J.I. Oñate, M. Brizuela, A. García-Luis, J.L. Viviente, F.J. García De Blas, A. Agüero, F. Longo y A. Roman, Proc. $8^{\text {th }}$ Int. Symp. Materials Space Environment, Arcachon, France, 2000, CNES (Eds.), Toulouse, Francia (2000) pp. 23-30.

[5] N.M. Renevier, M. Lobiondo, V.C. Fox, D.G. Teer y J. HAMPSHIRE, Surf. Coat. Technol. 123 (2000) 84-91.

[6] J.S. ZABINSKI, M.S. DONLEY, S.D. WALCK, T.R. SCHNEIDER y N.T. MCDEVITT, Tribol. Trans. 38 (1995) 894-904.

[7] D.G. Teer, J. Hampshire, V. Fox y V. Bellido-GonzÁlez, Surf. Coat. Technol. 94-95 (1997) 572-577.

[8] J. Serrano, J. Gómez-Elvira, R. Santiago, J. Pazos, J.I. Oñate, I. Garmendia y A. RodríGuez, SP-480, Proc. $9^{\text {th }}$ Eur. Space Mech. Tribol. Symp., Liège, Belgica, 2001, R.A. Harris (Ed.) ESA Public. Div. ESTEC, Keplerlaan 1. 2200 AG Noordwijk (NL), Liège, Belgium (2001) pp. 281-284.

[9] A. García, V. Bellido, N. Flaño y J.I. Oñate, Diamond and Related Materials 1 (1992) 350-354.

[10] M.A. Baker, R. Gilmore, C. Lenardi y W. Gissler, Appl. Surf. Sci. 150 (1999) 255-262.

[11] G. Muñiz, A. Conde, B.J. Fernández, R. Varela, I. García, J. De Damborenea. Rev. Metal. Madrid, 39 (2003) 443-451. 
Tribología de nuevas capas autolubricantes producidas mediante PVD J.I. Oñate, M. Brizuela, A. García-Luis, I. Braceras, S. Pachecho y J.L. Viviente

[12] E.W. RoberTs (ed.), Space Tribology Handbook, AEA Technology, 1997, Section 4.2.

[13] P. Niederhauser, H.E. Hintermann y M. Maillat, Thin Solid Films 108 (1983) 209-218.

[14] T. Spalvins, Thin Solid Films 118 (1984) 375-384.

[15] M.R. Hilton, R. Bauer, S.V. Didziulis, M.T. Dugger, J. Keem y J. Scholhamer, Surf. Coat. Technol. 53 (1992) $13-23$.
[16] N.M. Renevier, V.C. Fox, D.G. Teer y J. Hampshire, Surf. Coat. Technol. 127 (2000) 24-37.

[17] Matsumoto y M. Suzuki, Proc. 8th European Space Mechanisms Tribology Symp., Toulouse, France, 1999, D. Danesy (Ed.), ESA Public. Div. ESTEC, Noordwijk, NL (1999) pp. 43-48.

[18] W. Lauwerens, J. Wang, J. Navratil, E. WieËrs, J. D’HaEn, L.M. Stals, J.P. Celis e Y. BRuYNSERAEde, Surf. Coat. Technol. 131 (2000) 216-222. 